\title{
LUNGENEMBOLIE
}

\section{Jeder zweite Patient müsste nicht in die Klinik}

Eine Lungenembolie wird für gewöhnlich zunächst stationär behandelt. Möglicherweise ist die Klinikaufnahme aber viel seltener nötig als bisher angenommen, glauben die Autoren der europäischen Hestia-Studie. Mithilfe eines einfachen Fragebogens, dem „Hestia-Tool“, identifizierten sie aus einem Kollektiv von $581 \mathrm{~Pa}$ tienten mit akuter Lungenembolie solche mit geringem Risiko. Diese 297 Patienten wurden innerhalb von 24 Stunden nach der Diagnose mit einem niedermolekularen Heparin und einem Vitamin-K-Anta- gonisten nach Hause geschickt. Nach drei Monaten hatten nur $2 \%$ eine venöse Thrombembolie entwickelt. Eine Vergleichsgruppe fehlte, die Autoren betonen jedoch, dass die Erfolgsquote auf demselben Level wie bei stationär behandelten Patienten lag. Die Hestia-Kriterien beinhalten elf Fragen, u. a. zur hämodynamischen Stabilität, zur Notwendigkeit einer Thrombolyse oder Embolektomie sowie zum Blutungsrisiko.

American Society of Hematology 2010 Annual meeting; 7. 12. 2010: Orlando, FL. Abstract LBA-1

\section{RISIKOFAKTOR FLEISCHKONSUM}

\section{Schlaganfall vom Steak?}

Frauen, die viel rotes Fleisch essen, haben ein erhöhtes Schlaganfallrisiko. Im Rahmen der Swedish Mammography Cohort Study waren 34670 Teilnehmerinnen im Alter zwischen 49 und 83 Jahren nach ihren Ernährungsgewohnheiten befragt und zehn Jahre lang beobachtet worden. Frauen, die über $102 \mathrm{~g}$ rotes Fleisch pro Tag verzehrten, hatten im Vergleich zu denje- nigen, die weniger als $25 \mathrm{~g}$ täglich zu sich nahmen, ein um $42 \%$ höheres Risiko für einen Schlaganfall. Bereits in früheren Studien war gezeigt worden, dass Fleischesser öfter unter Hochdruck leiden als Vegetarier. Auch der hohe Eisengehalt von rotem Fleisch könnte für das Schlaganfallrisiko eine Rolle spielen.

Stroke 2010;DOI:10.1161/STROKEAHA.110.596510

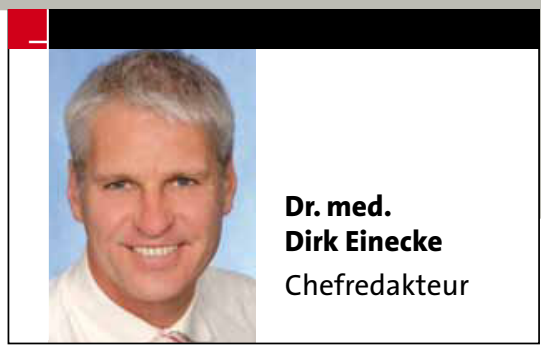

\section{KOLOSKOPIE}

\section{Krebsschutz nicht nur links}

Die Befürchtung, dass durch die endoskopische Untersuchung des Dickdarms Krebsvorstufen nur im Colon descendens zuverlässig entdeckt, im rechten Teil des Darms jedoch häufig übersehen werden, ist nicht berechtigt. Wissenschaftler vom Deutschen Krebsforschungszentrum hatten 1688 Darmkrebspatienten und 1932 Gesunde nach Koloskopien und dabei erhobene Befunde in den letzten zehn Jahren befragt. Insgesamt errechnete sich eine Senkung des Karzinomrisikos um $77 \%$, wenn im Zuge der Koloskopie Krebsvorstufen sofort entfernt wurden. Für den linken Dickdarm lag der Krebsschutz bei $84 \%$, aber auch für den rechten Darmabschnitt betrug die Risikoreduktion noch über 50\%. Bei alleiniger Sigmoidoskopie wären Krebsvorstufen in diesem Bereich übersehen worden.

Annals of Internal Medicine 2011; 154:22-30

\section{LEITLINIEN ZU OFT MISSACHTET}

\section{Tod nach ICD-Implantation}

Fast jedes vierte ICD-Implantat entspricht nicht den Empfehlungen der internationalen Kardiologen-Gesellschaften. Eine retrospektive Studie nutzte Datensätze eines US-Registers (NCDR) über 111707 Patienten, die zwischen 2006 und 2009 einen Cardioverter-Defibrillator erhalten hatten. In 22,5\% der Fälle waren die Leitlinien zur Implantation nicht genau befolgt worden. So waren in 24861 Fällen die Geräte deutlich zu früh eingepflanzt worden (die Leitlinien sehen einen Abstand von mindestens drei Monaten nach einer Herzinsuffizienzdiagnose bzw. von 40 Tagen nach einem Myokardinfarkt vor). 3022 Patienten hätten überdies aufgrund ihrer Symptome (NYHA-Klasse IV) gar keinen ICD erhalten dürfen. Die Missachtung der Empfehlungen hatte gravierende Folgen: Verglichen mit einem Kollektiv, bei dem die Prozedur leitliniengerecht erfolgte, verstarben die betroffenen Patienten signifikant häufiger noch in der Klinik (0,57\% vs. 0,18\%) bzw. erlitten öfter Komplikationen (3,23\% vs. 2,41\%).

JAMA 2011;305:43-49

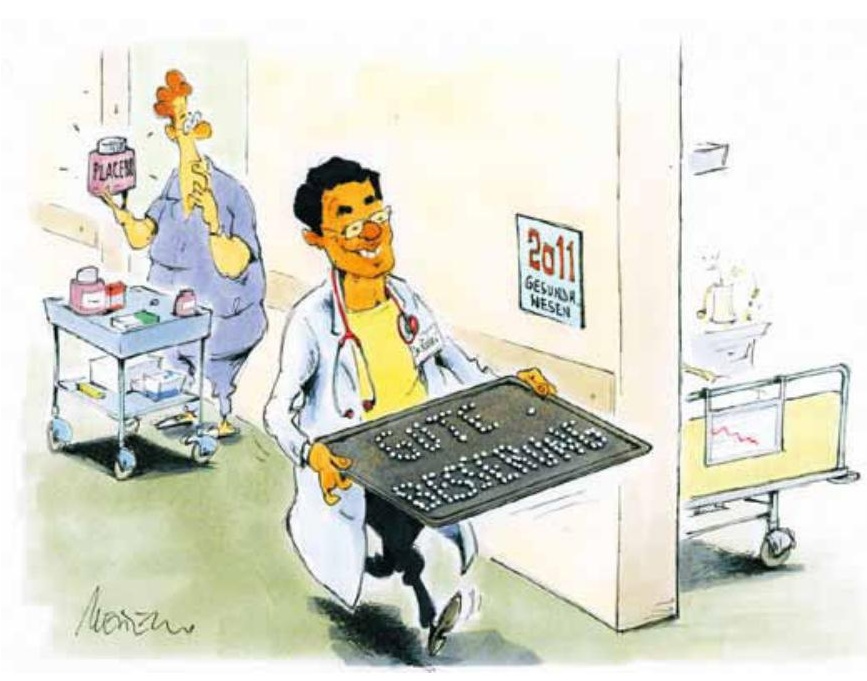

